UCRL-JC-120743

PREPRINT

\title{
Design of a Mid-IR Immersion Echelle Grating Spectrograph for Remote Sensing
}

Norman L. Thomas, Isabella T. Lewis, and Charles G. Stevens

This paper was prepared for submittal to the SPIE Aerosense 1995 Conference, Orlando, Florida April 17-22, 1995

May 9, 1995

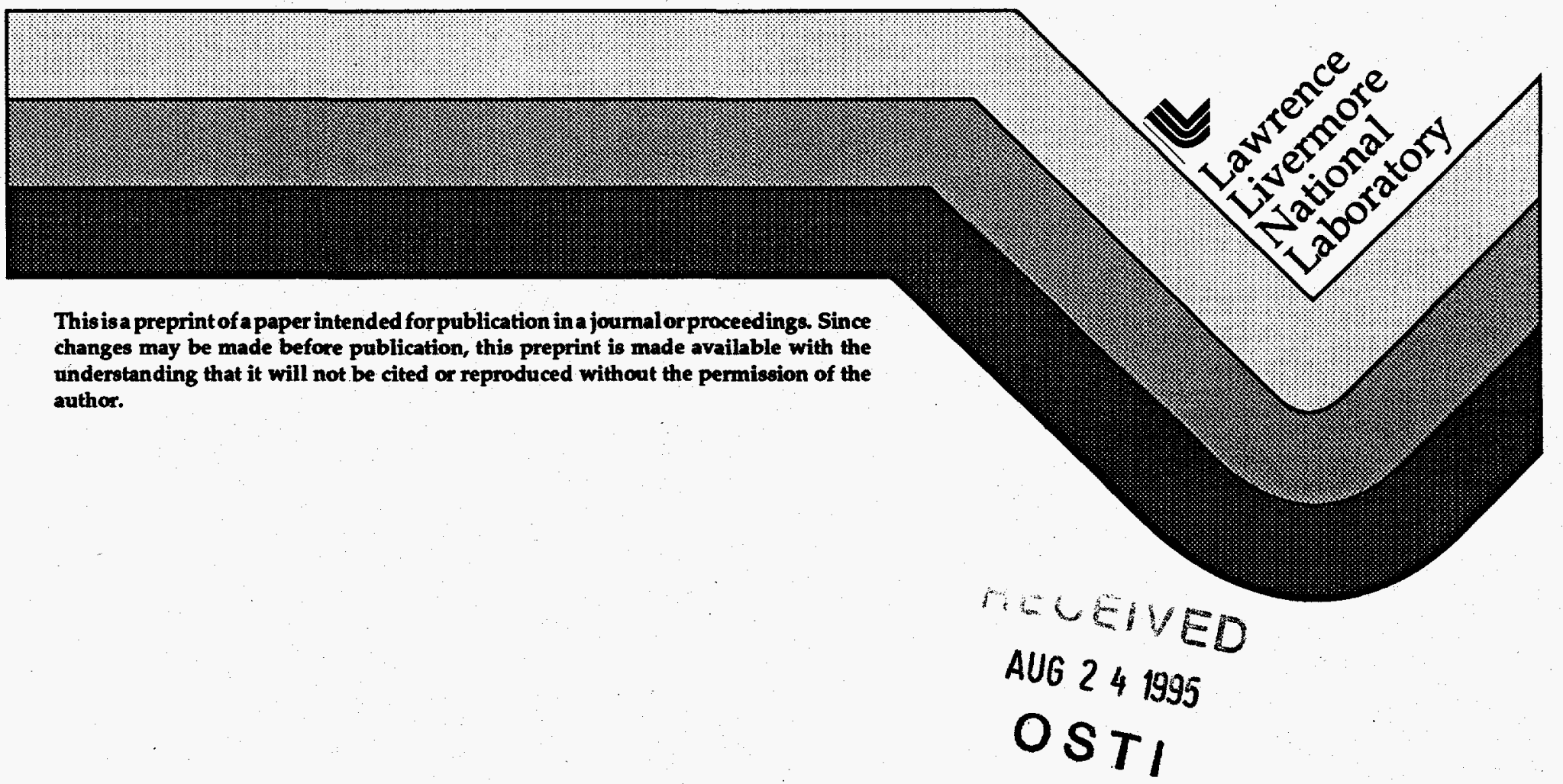




\section{DISCLAIMER}

This document was prepared as an account of work sponsored by an agency of the United States Government. Neither the United States Government nor the University of California nor any of their employees, makes any warranty, express or implied, or assumes any legal liability or responsibility for the accuracy, completeness, or usefulness of any information, apparatus, product, or process disclosed, or represents that its use would not infringe privately owned rights. Reference herein to any specific commercial product, process, or service by trade name, trademark, manufacturer, or otherwise, does not necessarily constitute or imply its endorsement, recommendation, or favoring by the United States Government or the University of California. The views and opinions of authors expressed hereir do not necessarily state or reflect those of the United States Government or the University of California, and shall not be used for advertising or product endorsement purposes. 


\section{DISCLAIMER}

Portions of this document may be illegible in electronic image products. Images are produced from the best available original document. 
Design of a mid-IR immersion echelle grating spectrograph
for remote sensing

\author{
Norman L. Thomas, Isabella T. Lewis \\ and Charles G. Stevens
}

Lawrence Livermore National Laboratory

P.O. Box 808

Livermore, California 94550

\begin{abstract}
We describe the design of a silicon immersion grating spectrograph for the remote detection of chemicals in the atmosphere. The instrument is designed to operate in the two atmospheric windows from 2.3 to 2.5 and 2.8 to 4.2 microns at a resolution of $0.1 \mathrm{~cm}^{-1}$. This is achieved by cross dispersing a high order silicon immersion echelle $(13.5$ grooves $/ \mathrm{mm})$ and a first order concave grating operating in a reflective configuration to generate a two-dimensional spectrum in the image plane with diffraction limited performance.
\end{abstract}

\title{
1. INTRODUCTION
}

The remote detection of airborne chemicals issuing from exhaust stacks offers a non-intrusive means for monitoring and attributing pollution source terms. To detect, identify and quantify a chemical effluent, it is highly desirable to operate at the limiting spectral resolution set by atmospheric pressure broadening at approximately $0.1 \mathrm{~cm}^{-1}$. This provides for maximum sensitivity to simple molecules with the narrowest spectral features, allows for corrections for the presence of atmospheric constituents, maximizing species selectivity, and provides greater opportunity to detect unanticipated species. Fourier transform spectrometers have been the instrument of choice in the infrared because of their potential for high resolution, high signal to noise and broad spectral coverage. However, several inherent features limit their performance when observing localized sources from moving platforms: the precision requirements of the moving interferometric mirror can limit the achievable resolution in field settings, the intolerance to temporal fluctuations in the observed scene creates noise problems when mounted on airborne platforms viewing rapidly changing ground terrain and finally, they are limited in ultimate signal-to-noise performance by the use of spectral multiplexing. Recent advances in two-dimensional infrared detector array capacity and performance along with advances in chemical micro-machining technology ${ }^{1}$ provide the opportunity for creating a new class of remote sensing IR spectrometers. The design we will describe is the second generation of remote sensing echelle grating spectrometers developed at the Lawrence Livermore National Laboratory and the second design based on the availability of silicon immersion gratings of high precision. The dispersion of an immersion grating is increased in proportion to the refractive index. For silicon this factor is 3.4, permitting a very significant reduction in the overall size of the spectrometer while maintaining the same resolution and light throughput. The objective of the design has been to develop a spectrometer covering the MWIR atmospheric windows with no moving components by using a cross dispersion approach. Our earlier designs 2,3 utilized an echelle grating 
crossed with a constant dispersion prism doublet. This provided for very efficient detector array utilization. However, with the smaller $(\times 3.4)$ immersion spectrometer, the need to increase prism dispersion forced a number of design compromises in overall size and system performance. The design presented here is based on the use of a high order silicon immersion grating crossed with a concave grating operating in a Wadsworth configuration. The design approach taken to achieve diffraction limited performance is described.

\section{DESCRIPTION}

The instrument design, shown in Fig. 1 (vertical view) and Fig. 2 (horizontal view), consists of a dual fiber bundle input for the 2.33-2.48 and 2.82-4.17 micron regions, hereafter referred to to as the lower and upper bands, respectively. Short wave pass and long wave pass filters are used to separate the lower and upper bands in the fiber output beams. The fiber bundles are $\mathrm{ZrF}$ fibers that couple to the output of a telescope and are formed into a linear array to become the entrance slit of the spectrometer. The entrance slits are 100 microns wide and 400 microns in length which transmit the IR radiation into two $f / 8$ cones entering the spectrometer. The two separate fiber bundles are separated by $8 \mathrm{~mm}$, or $1.75^{\circ}$ field angle, at the focus of the collimating parabola. This offset results in displacements of the echellogram fields at the final focus of the spectrometer and aligns $2.48 \mu \mathrm{m}$ from the short wave fiber to $2.82 \mu \mathrm{m}$ from the long wave fiber. The focal plane thus covers the wavelength range from 2.33 to $2.44 \mu \mathrm{m}$ and 2.82 to $4.17 \mu \mathrm{m}$, eliminating the rows that would otherwise be reserved for information from the water band, which is unusable due to atmospheric absorption. Signal overlap between the fiber inputs is avoided by the use of bandpass filters on each fiber output. Net cross talk at the focal plane between the overlapping fiber inputs is less than $1 \%$.

The fiber optic inputs are collimated by a parabola, which is used off-center and off-axis, with two anti-symmetric, weak cylinders located after each fiber optic bundle. Mechanical packaging and field angle considerations require an $8 \mathrm{~mm}$ separation between the fibers. Without correction, the aberration between the two fibers, each at best focus, would be $0.095 \mathrm{rms}$ astigmatism at the working wavelength, for a $200 \mu \mathrm{rad}$ blur, which is not correctable in the remainder of the imaging path. The uncorrected spotsize is shown in Fig. 3a. This is equivalent to a $20 \mu$ blur in the focal plane. The addition of the cylinder lenses corrects all astigmatism aberrations, leaving a net residual $0.006 \mathrm{rms}$ coma at the working wavelength, equivalent to $40 \mu \mathrm{rad}$, or $4 \mu$ at the focal plane. The corrected spotsize is shown in Fig. $3 \mathrm{~b}$.

The first disperser is a silicon immersion echelle grating with a spatial frequency of 13.5 grooves $/ \mathrm{mm}$ and a blaze angle of 63.5 degrees (along the $x$-axis out of the plane in Fig. 2). The immersion echelle operating in high order disperses along the the $\mathrm{x}$-axis orthogonal to the $\mathrm{y}$-axis (concave grating dispersion direction). When the grating is immersed in a refractive medium, the resolving power and dispersion are increased by the value of the index, and the free spectral range is decreased by the same factor. The refractive index of silicon is 3.4 which increases the dispersion by 3.4 and allows the instrument to be reduced in size compared to conventional grating spectrographs. Once the spectrograph is reduced in size, the concave grating (order sorter) dispersion must be increased proportionately to maintain the required dispersion in the detector plane. This is easily accomplished by increasing the grating frequency of the concave grating. 
The concave grating is used instead of a plane grating to add optical power to the optical train so that the beam does not expand excessively before entering the condensing lens. The radius of the concave grating is $750 \mathrm{~mm}$ and the grating frequency is 85 grooves $/ \mathrm{mm}$. Several grating radii of curvature $(0.5$, $0.75,1$ meter radii as well as flat) were examined, trading image aberrations in the image plane against beam size at the condensing lens location. Longer radius gratings introduce less image aberration but the size of the emerging beam as it clears the incoming beam can be excessive. A flat grating presents a dispersing beam size of $125 \mathrm{~mm}$ at the lens location. A suitable trade-off has been found with a 0.75 meter radius grating, providing a $40 \mathrm{~mm}$ beam and an aberration spot size less than the diffraction limit.

After passing through the crossed-dispersers in a single pass, the beam is focused at the detector plane with the condensing lens. The condensing lens is a silicon/germanium/silicon triplet achromatized for the wavelength range 2.3-4.2 microns. At the detector plane, two wavelengths separated by $0.1 \mathrm{~cm}^{-1}$ are just resolved in the echelle grating dispersion direction (x-axis). The magnification of the system is 0.37 and a $100 \times 400$ micron slit maps onto a $38 \times 148$ micron image plus small aberrations and diffraction, the system being diffraction limited. In the image plane, the spectrum dimensions are $12.8 \times 12.8 \mathrm{~mm}^{2}$. A cold shield is located before the final silicon lens, both of which are cooled to 50 degrees Kelvin. This cold shield limits the thermal radiation seen by the detector elements and reduces noise. The IR spectrograph components and fixtures are cooled to 150 degrees Kelvin in order to further reduce the IR background and improve the sensitivity. Since the fixtures, mounting plate, parabolic mirror (diamondturned), and concave grating are made of aluminum, the entire mounting system shrinks uniformly as it is cooled and no severe distortions or increase in aberrations occurs. The design is based on dispersion data calculated at low temperature using OPTIMATR (ARSoftware) and thermal expansion coefficients from the literature. The dispersion data is calculated using the Sellmeier equation for low temperatures.

The echellogram is designed to fit on a $512 \times 512$ array detector $(12.8 \times 12.8 \mathrm{~mm}$ square $)$ having a 25 micron pixel size. This is depicted in Fig. 8. Also shown is the size of a smaller $256 \times 256$ array detector $(9.72 \times 9.72 \mathrm{~mm}$ square $)$ having a 31 micron pixel size.

\section{DESIGN GOALS}

The design goals included covering the detector array with the wavelength range 2.33 to 4.17 microns (except for the water band). Shown below are the overall design parameters for the instrument.

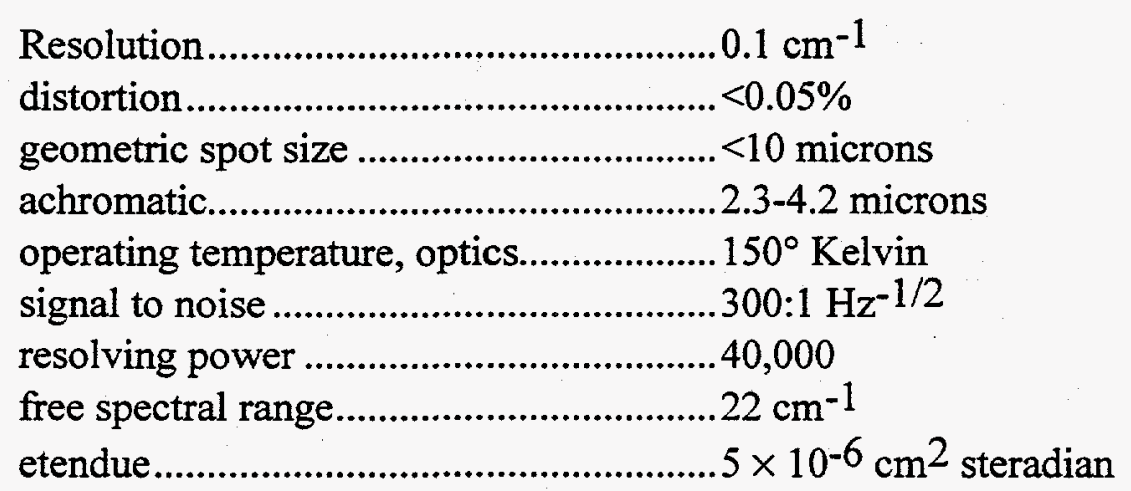


The instrument was designed with no moving components. The optical components are mounted without the need for external adjustments during operation. The instrument will be housed in an evacuated enclosure during deployment.

\section{ECHELLE IMMERSION GRATING}

The echelle immersion grating frequency is 13.5 grooves $/ \mathrm{mm}$ and is located on the second surface of a silicon prism, the grating surface being tilted away from the optic axis by 63.5 degrees. The echelle orders range from 108 to 159 for the upper band and 181 to 194 in the lower band, for a total of 66 orders. The echelle grating is depicted in Fig. 4 with the entrance face normal to the optic axis. The presence of the refracting medium increases the resolving power and dispersion of the grating 4 by a factor equal to the index of refraction and decreases the free spectral range by the same factor. In order to maintain the same free spectral range $\left(22 \mathrm{~cm}^{-1}\right)$, the step height, $\mathrm{d}$, in the direction of the optic axis can be decreased by the index of silicon, a factor of 3.4. Gratings made by conventional ruling engines are available with frequencies as low as 23 grooves $/ \mathrm{mm}$ whereas this design requires 13.5 grooves $/ \mathrm{mm}$. The development of this grating is described in another paper ${ }^{1}$.

\section{CONCAVE GRATING}

The concave cross-dispersing grating is a 85 grooves $/ \mathrm{mm}$ replicated grating on an aluminum substrate with a radius of curvature of 0.75 meter. Although $0.5,0.75$ and 1 meter radii were examined for overall performance, the 0.75 radius gave the minimum aberrations when used in the Wadsworth configuration;i.e., the exiting beam is perpendicular to the grating on center. When the spherical grating is used off-axis, it introduces coma and astigmatism in addition to the spherical aberration. Code V was used to optimize both the concave grating and the triplet condensing lens to give geometric spot sizes between 5 and 10 microns diameter. The use of the concave grating instead of prisms as used in an earlier design 2 eliminated the curvature and distortion of the bands in the echellogram.

\section{CONDENSING LENS}

The condensing lens, in conjunction with the curved cross-disperser grating, serves to image the collimated light spectral orders from the echelle grating onto a $12.8 \mathrm{~mm} \times 12.8 \mathrm{~mm}$ array. The condenser lens consists of three lenses of silicon and germanium which have an effective focal length of $70 \mathrm{~mm}$. The first element is a positive silicon lens which is combined with a negative germanium lens to form an achromatic doublet. The third lens is a positive germanium lens which acts as a field lens, helping to correct some of the distortion introduced by the concave grating. The exiting cone of light is $f / 3$ giving diffraction spot sizes from 18 to 30 microns diameter over the wavelength range 2.33 to 4.17 microns, respectively. The geometric spot sizes vary from 5 to 10 microns, providing diffraction limited performance. 
The silicon/germanium doublet is cooled to 150 degrees Kelvin while the cold stop and silicon field lens are cooled to 50 degrees Kelvin. The condenser lens is optimized to operate at these temperatures. A high efficiency cold stop was not required at these operating temperatures to prevent spectrometer component radiation from degrading sensitivity. Fig. 5 shows the design of the triplet in the $y-z$ plane.

Fig. 6 shows spot diagrams for some selected wavelengths in the lower and upper bands. These are superimposed over the echellogram which shows the spatial location of each selected spot in the detector plane. Most of the energy is concentrated in an area less than 10 microns diameter. Fig. 7 shows the diffraction intensity spread for the wavelength $3355 \mathrm{~nm}$.

Fig. 8 shows the echellogram for 15 wavelengths with the detector limits superimposed. Although the free spectral range is constant at $22 \mathrm{~cm}^{-1}$, the physical length of the horizontal bands changes from top to bottom since the dispersion of the echelle grating has a frequency dependence causing this contraction.

The abrupt change in width near the lower edge of the echellogram is due to the superposition of the two wavelength bands. Note that the vertical and horizontal dimensions of the diagram are well matched. The grating constant on the concave grating, which controls the vertical dispersion of the beam, is designed in conjunction with the echelle grating, which controls the horizontal dispersion of a free spectral range. Note that this echellogram shows only the dominant diffraction order. Wavelength repeats at the far left and right of the diagram. Since the concave grating cross-disperser, which is used only in first order, acts the same for the single wavelength split between the left and right edges, the height of these two images is identical. The crossed-grating system results in a slight slope with increasing wavelength with respect to the horizontal axis as defined by the grating surfaces. A slight rotation of the focal plne array about the optic axis will be made to adjust the echellogram rows parallel to the detector row axis.

\section{SPECTRAL ORDERS}

There are 14 lower spectral orders and 52 upper spectral orders. Fig. 9 shows the wavelength table including order numbers, edge and center wavelengths, and free spectral range. The band orders extend from 108 to 194 with orders 160 to 180 corresponding to the water band delibrately omitted. The wavelengths are expressed in nanometers.

\section{CONCLUSIONS}

We have described the design of a second generation remote chemical sensing echelle grating spectrometer under development at LLNL. The design is centered about the use of a coarse (13.5 grooves $/ \mathrm{mm}$ ) silicon immersion grating crossed with a first order concave order sorting grating to create a MWIR spectrometer with no moving components. The main design goals were to create a compact $(1 / 3$ meter) instrument with resolving power greater than 30,000. Small pre-correction lenslets (cylinder lenses) play a key role in reducing off-axis aberrations, providing diffraction limited performance.

\section{ACKNOWLEDGEMENTS}

*This work was performed under the auspices of the U.S. Department of Energy by Lawrence Livermore National Laboratory under contract No. W-7405-Eng-48. 


\section{REFERENCES}

1. Paul J. Kuzmenko, D.R. Ciarlo, and C.G. Stevens, "Fabrication and testing of a silicon immersion grating for infrared spectroscopy," Optics, Imaging, and Instrumentation, SPIE, Vol. 2266, 566-577 (1994).

2. Charles G. Stevens, Paul Kuzmenko, Terry W. Alger, and Norman L. Thomas, "An echelle grating spectrometer (EGS) for mid-IR remote chemical detection," Optics, Imaging, and Instrumentation, SPIE, Vol. 2266, 2-12 (1994).

3. Norman L. Thomas, Steve A. Johnson and Charles G. Stevens, "Design of an immersion echellegrating mid-IR spectrograph for chemical remote sensing" Optics, Imaging and Instrumentation, SPIE, Vol. 2266, 13-24 (1994).

4. E. Hulthen and H. Neuhaus,"Diffraction gratings in immersion", Arkiv fur Fysik, vol. 8, 343-353 (1954)

5. K.H. Hinkle, S.T. Ridgway and F.E. Stuart, "Optical design for a high resolution, 2-5 micron infrared cryogenic echelle spectrometer," Intrumentation in Astronomy, SPIE, Vol. 1235 (1990).

6. Daniel J. Schroeder, "Design considerations for astronomical echelle spectrographs," Pub. Astro. Soc. Pacific, 82, 1253 (1970). 


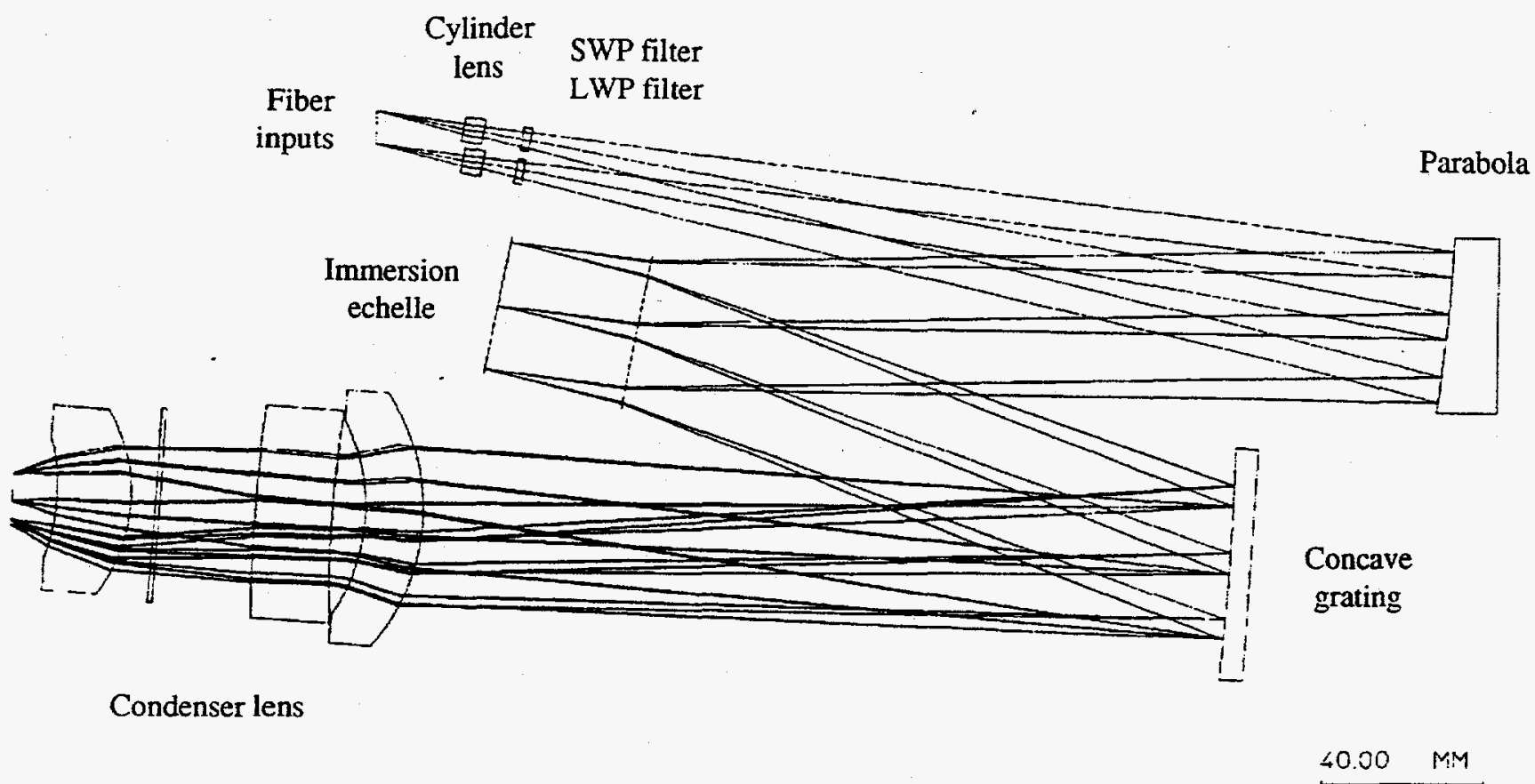

Figure 1. Vertical view of immersion echelle/grating spectrograph (y-z plane)

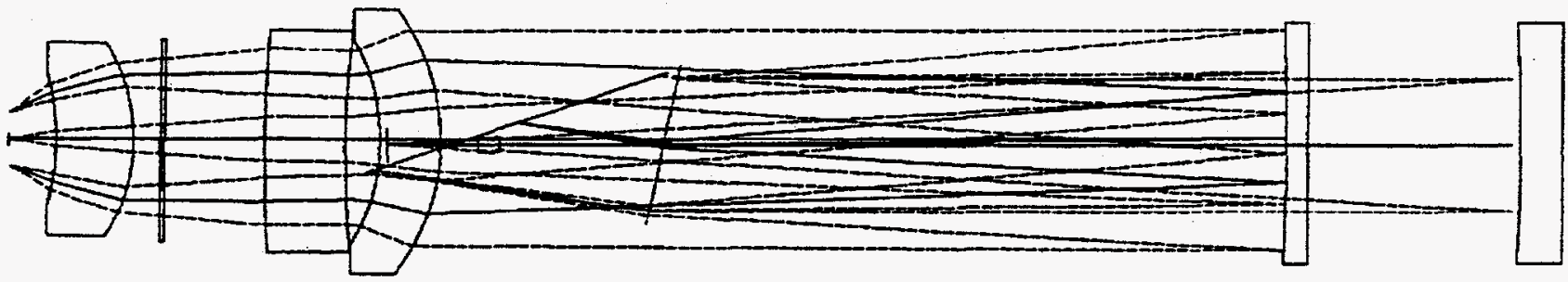

Figure 2. Horizontal view of immersion echelle/grating spectrograph ( $x-z$ plane) 


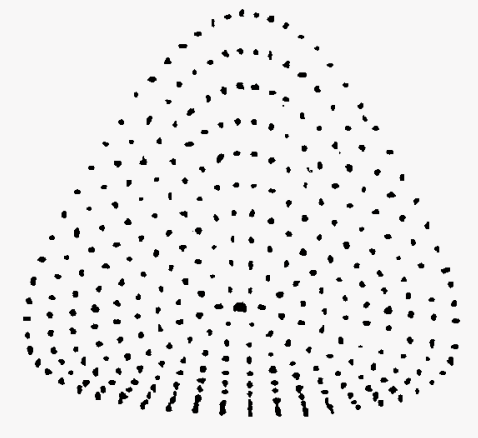

(a) uncorrected

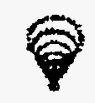

(b) corrected

$$
20 \mu \mathrm{m}
$$

Figure 3. Effect of cylinder lens on geometric spot size

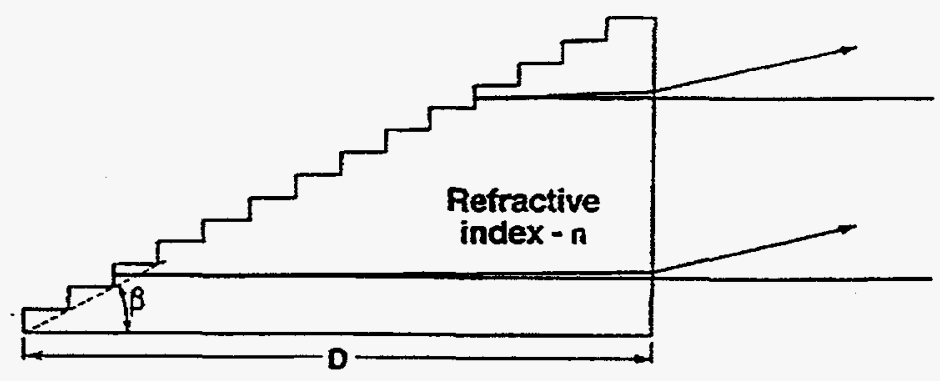

$$
\begin{aligned}
\text { Resolving power }(R) \frac{\lambda}{\Delta \lambda} & =\frac{n D}{1 / 2 \lambda} \\
\text { Linear dispersion } & =\frac{n f}{(1 / 2 \lambda) \tan \beta}
\end{aligned}
$$

Figure 4. Immersion echelle grating 


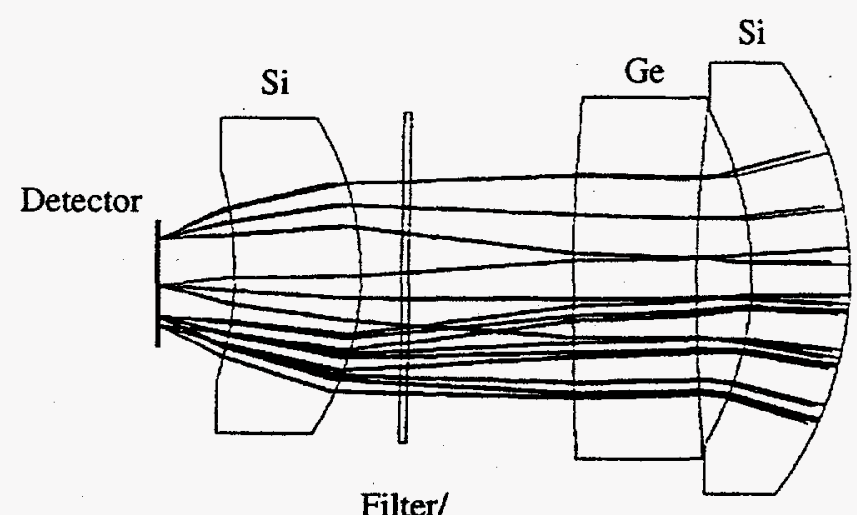

Filter/

cold shield

$\underline{500 \quad m}$

Figure 5. Silicon/germanium triplet condensing lens
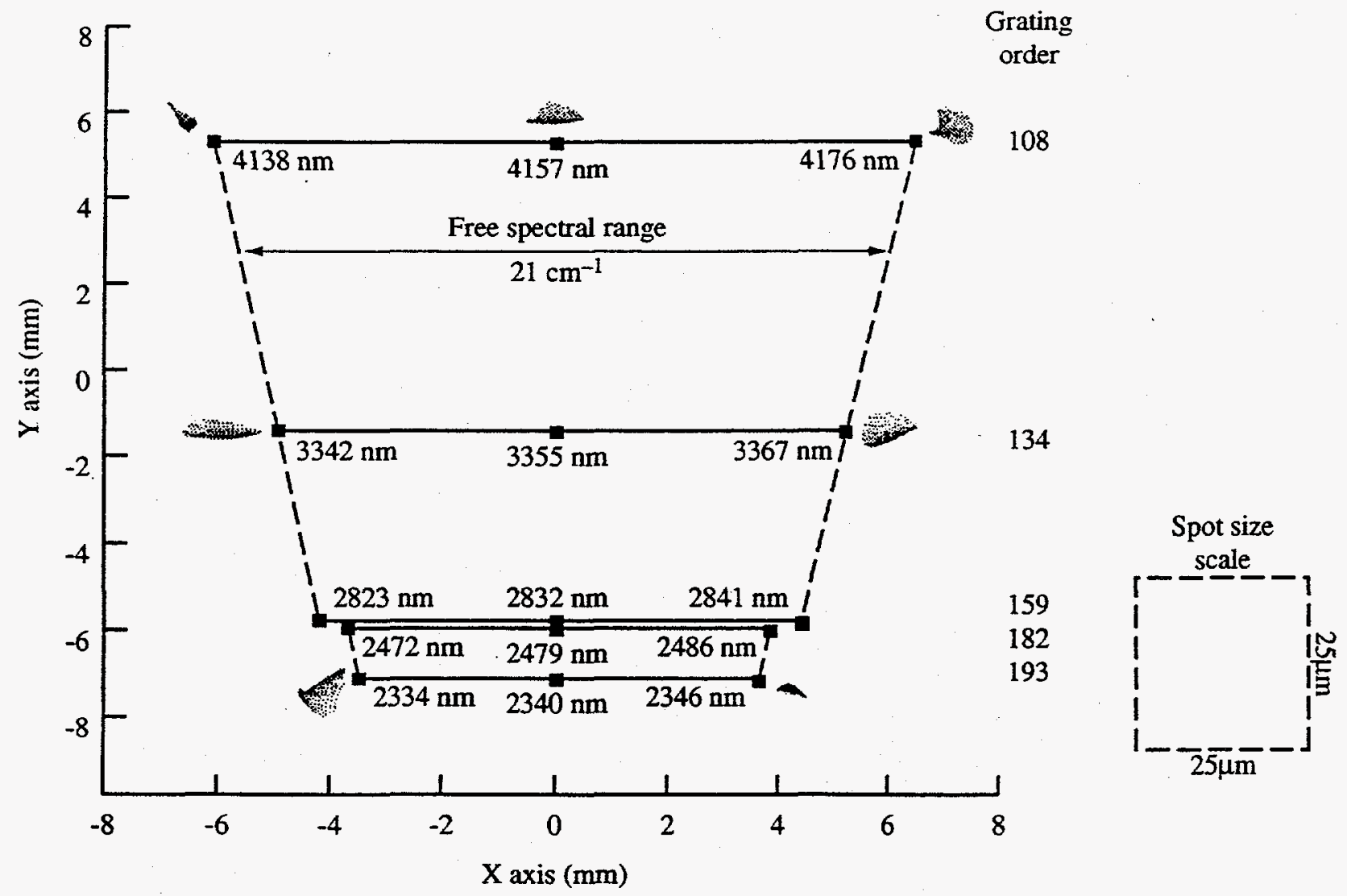

Figure 6. Spot diagrams for selected wavelengths in the upper and lower bands 


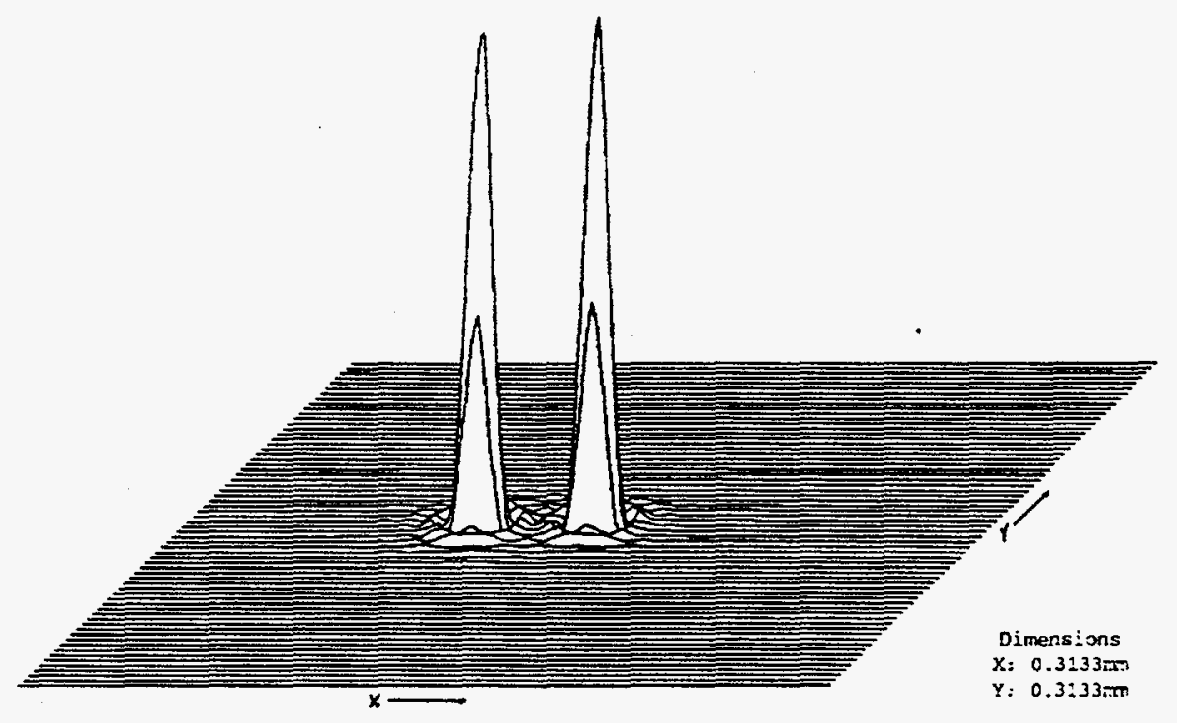

Figure 7. Diffraction intensity spread function for frequency pairs separated by $0.1 \mathrm{~cm}^{-1}$

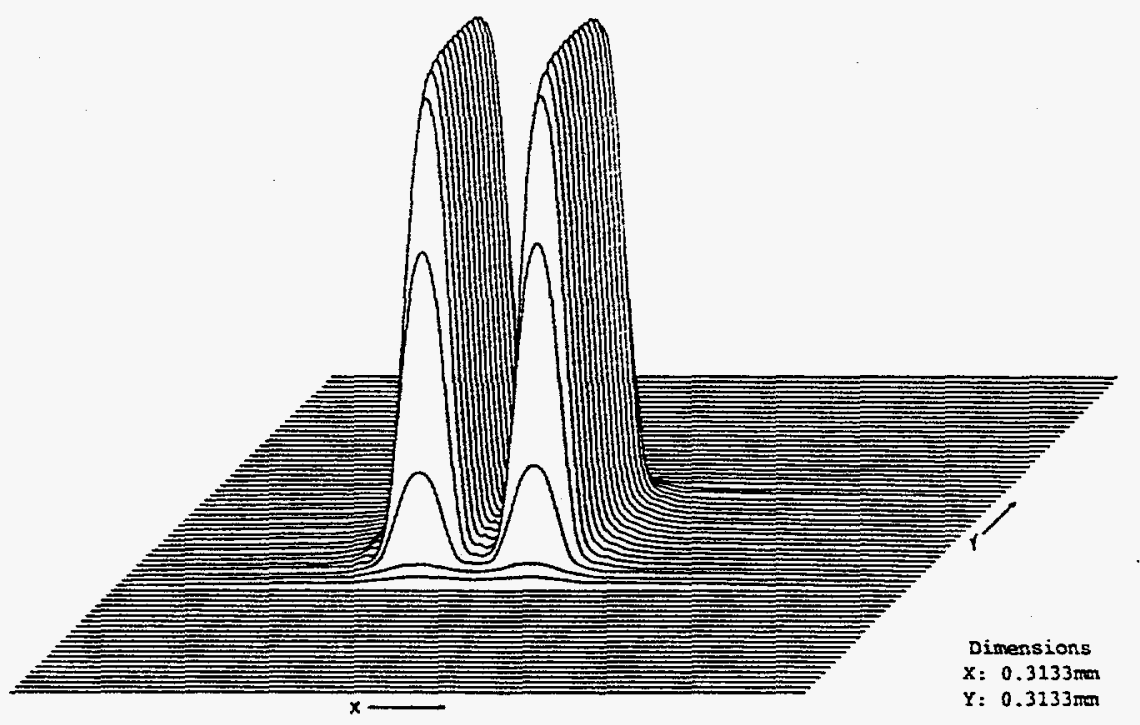

Figure 8. Image of $100 \times 400 \mu \mathrm{m}$ slit convolved with diffraction point spread functions for frequency pairs separated by $0.1 \mathrm{~cm}^{-1}$ 


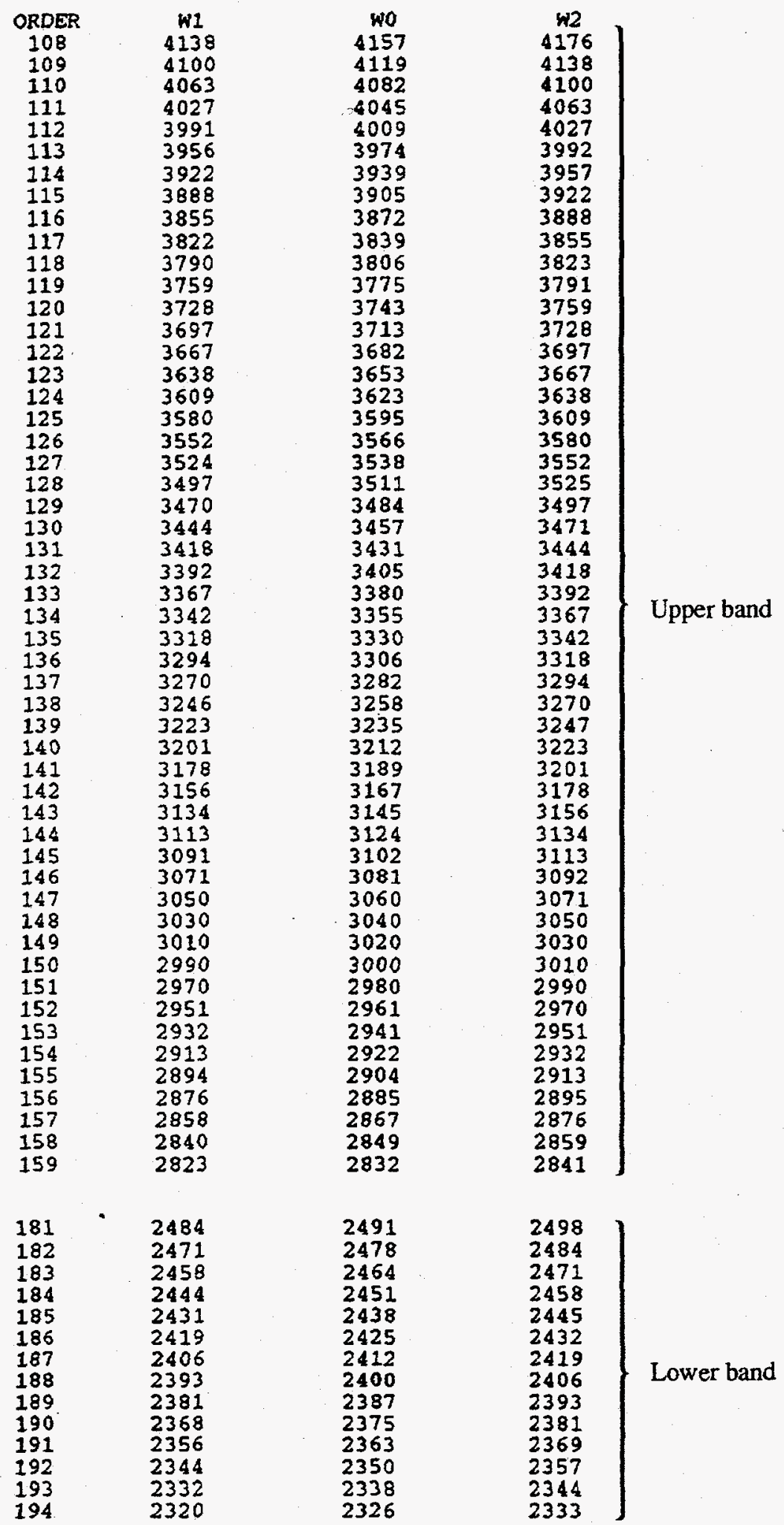

Figure 9. Wavelength table for upper and lower bands (nm) 